\title{
On Sensitivity of Cherenkov Radiation to the Dynamics of High Energy Cosmic Ray Interactions
}

\author{
J. Ř́́dký ${ }^{a}$ and D. Nosek ${ }^{\mathrm{b}} \dagger$ \\ anstitute of Physics of the Academy of Sciences of the Czech Republic, \\ Na Slovance 2, 18221 Prague 8, Czech Republic \\ b Institute of Particle and Nuclear Physics, Charles University, \\ V Holešovičkách 2, 18000 Prague 8, Czech Republic
}

A simulation study of the effects resulting from creation of quark-gluon plasma in high energy collisions of cosmic iron nuclei with the air has been carried out. The Cherenkov light emission has been found to reveal some features of the dynamics of high energy hadronic interactions.

\section{Introduction}

One possible way to investigate rare ultraenergetic collisions of cosmic ray primaries with the air is to investigate the Cherenkov light emitted in atmospheric showers. Charged daughter particles produced along a hadronic cascade have mostly very high velocities and therefore emit in the air Cherenkov photons which in principle carry an information about longitudinal shower development including an information on the characteristics of the primary collision, e.g. particle multiplicity. As optical photons suffer relatively smaller absorption in the atmosphere, their density on the ground is higher than that of charged particles and their lateral distribution is broader. The Cherenkov light highlights differences in the mechanism of hadronic collisions that are expected to occur in the first stage of the shower development and that can propagate further during development of the cascade.

\section{Primary Interaction}

Since available experimental data do not contradict to the fact that the abundance of ${ }^{56} \mathrm{Fe}$ nuclei in the primary flux may be above $10 \%$ [1], a non-negligible portion of iron-nitrogen or ironoxygen nucleus-nucleus collisions can be observed

\footnotetext{
*ridky@fzu.cz

${ }^{\dagger}$ nosek@ipnp.troja.mff.cuni.cz
}

in upper atmosphere. The number of nucleons participating in such collisions may well grow up to a few tens and a fireball of hot and dense elementary matter of quarks and gluons (QGP) can be created. Its thermal equilibrium is achieved in rapid statistical energy distribution among partons. As a result an almost ideally thermalized system of nucleons, pions and kaons (heavier baryons and mesons are neglected in our approach) is produced in the primary collision.

The energy density of the fireball can be estimated from the Bjørken formula [3], $\epsilon=\frac{1}{\pi R^{2} \tau} \frac{\Delta E}{\Delta y}$, where $R$ is the radius of the smaller nucleus, the formation time was chosen as $\tau \approx 1 \mathrm{fm} / \mathrm{c}$ and $\Delta E$ is c.m.s. energy of produced particles in the unit rapidity interval in the vicinity of its c.m.s. value. Energy densities and corresponding upper bounds of the transition temperatures achieved in our simulations of the primary interaction of the cosmic iron nucleus with the air nitrogen are shown in Fig 1 as functions of the Fermi energy variable $F \approx\left(s_{N N}\right)^{\frac{1}{4}}$, where $\sqrt{s_{N N}}$ is an invariant mass of a pair of interacting nucleons 2].

When the number of wounded nucleons $N_{\text {Int }}$ in the first collision is greater than a certain value (typically $N_{\text {Int }} \geq 20$ ), we assume that several nucleons melt down into the QGP phase and a certain part of the total mass of the fireball $E_{\mathrm{hot}} \approx \eta \sqrt{s_{\mathrm{F}}}$ is released. The model parameter $\eta$ $(\eta=0.1-0.4)$ is assumed to be independent of 
the collision energy and the system size. A portion of released energy is channeled to nucleons with momenta generated according to the relativistic Boltzmann distribution isotropically with respect to the fireball center of mass system. The remaining energy is then used to create a number of $\pi$ and $K$ mesons with isotropic and relativistic Boltzmann distributed momenta in fireball system. Thermalized hadronic species are produced with probabilities $N: \pi: K \approx 10: 85: 5$ and with approximately equal abundances of different charges. Chemical freeze-out is controlled by a constant temperature $(k T=170,340 \mathrm{MeV})$ and zero chemical potentials. Multiplicities of light mesons produced in the initial interaction, as calculated in our model, are shown in Fig 2

\section{Shower Simulations}

To achieve the quantitative results, the CORSIKA simulation code [4 has been adapted to calculate the contributions of the Cherenkov photon intensity stemming from the quark-gluon equilibration in primary collisions of the cosmic iron nuclei with the air. The QGSJET01 model of high energy interactions has been employed; the GEISHA procedure has been used to treat hadronic collisions at energies $E<80 \mathrm{GeV}$. It is worth to note that the uncertainty related to the modelling of standard hadronic interactions turns out to be of minor influence, as the present investigation of QGP effects relies upon comparative characteristics of the detectable photons.

We analyze the contributions of Cherenkov light to the lateral ground level distribution functions of iron-induced showers for a given primary energy $\left(E_{\mathrm{Fe}}=100 \mathrm{TeV}-10 \mathrm{EeV}\right)$ averaged over 100 showers. The primary zenith angle was fixed at zero degrees; the altitude of the initial interaction was left free. The kinetic energy cutoffs for hadrons and muons have been set to $0.3 \mathrm{GeV}$; for electrons/positrons and photons we use cutoffs of $20 \mathrm{MeV}$ and $2 \mathrm{MeV}$, respectively. In all calculations a thinning level $10^{-7}$ has been adopted. The Cherenkov light has been collected by a row of 100 ideal detectors with detection area $3 \times 3 \mathrm{~m}^{2}$ and spacing $10 \mathrm{~m}$ located in north-south direction at a height of $1 \mathrm{~km}$ above the sea level $\left(813 \mathrm{~g} \cdot \mathrm{cm}^{-2}\right)$.
In all simulations the standard U.S. atmosphere has been used; propagation of the Cherenkov light has been corrected for attenuation in the air.

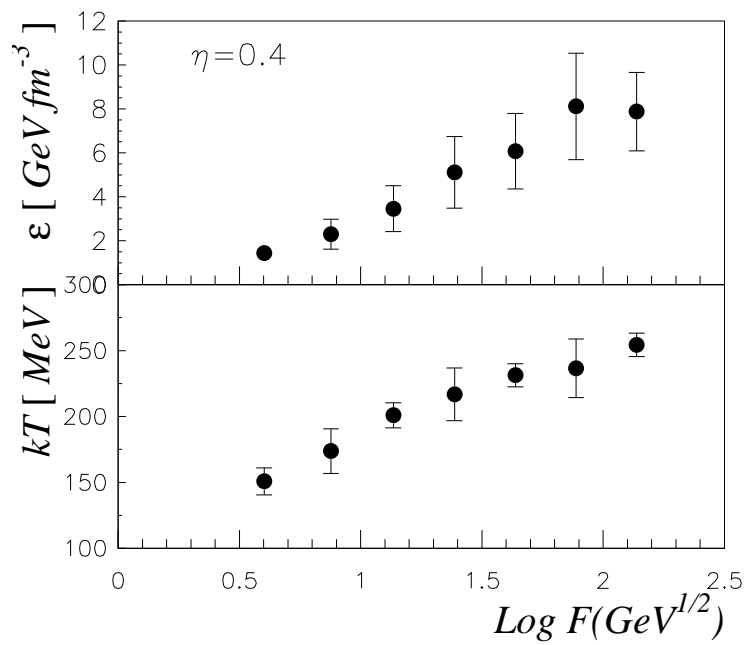

Figure 1. Estimated energy densities and transition temperatures achieved at the first $\mathrm{Fe}-\mathrm{N}$ interaction are depicted as functions of the Fermi energy variable (see text).

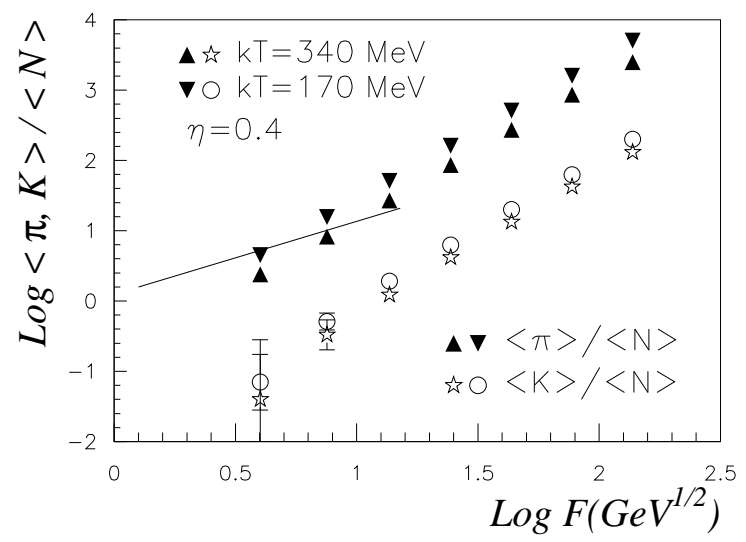

Figure 2. The average yields of pions and kaons per average number of participating nucleons as functions of the Fermi energy variable. SPS and RHIC experimental results for pions are indicated by the straight line.

\section{Effects of QGP formation}

In our simple model, more secondary particles are created in the first interaction when QGP is formed (QGP shower) compared to the ordinary $\mathrm{Fe}-\mathrm{N}$ collisions (Fe shower). Air showers following QGP production have their maxima higher 
in the atmosphere and produce noticeably more Cherenkov light at higher altitudes; this effect grows with increasing incident energy. In Fig 3

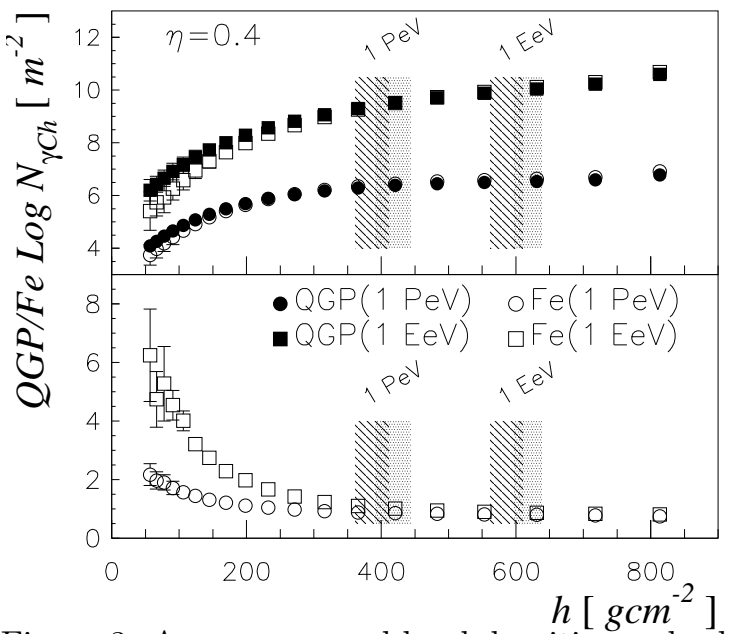

Figure 3. Average ground level densities and relative QGP/Fe numbers of Cherenkov photons are depicted as functions of the slant depth of their production. Hatched (dotted) boxes correspond to maxima of the QGP (Fe) showers.

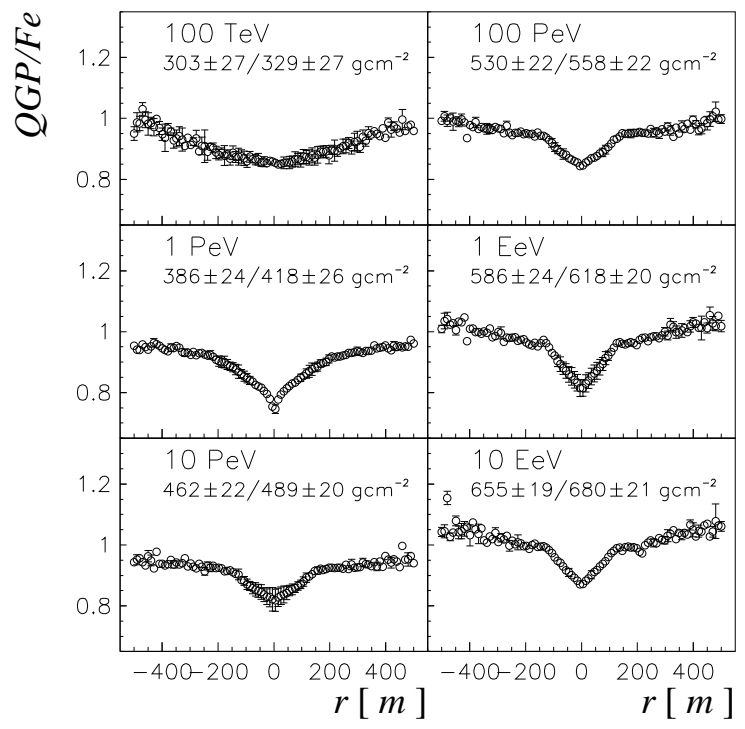

Figure 4. Relative QGP/Fe ground level lateral distribution of Cherenkov photons. Average values of QGP and Fe shower maxima are indicated.

the average ground level densities and ratios of total numbers of Cherenkov photons produced along the QGP and Fe showers are depicted as functions of the slant depth of their birth.
Average ratios of the ground level Cherenkov photon lateral densities from the QGP and Fe air cascades are depicted in Fig 4 The photon core densities of the QGP showers are typically by a factor of 1.2 smaller than the core densities of the Fe showers of the same primary energy. At very high primary energies, $E_{\mathrm{Fe}} \geq 1 \mathrm{EeV}$, the relative fraction of photons at outer parts of the QGP shower increases. Thus the QGP effects could be visible comparing the number of Cherenkov photons reaching the detector level relatively far from the shower core with all detected photons.

Any influence of the transverse momenta of the direct hadrons in the first interaction becomes negligible as in the case of very energetic primary the lateral profile of shower is dominated by interactions of particles at lower energies in the later part of the shower development. The QGP showers behave similarly as ordinary hadronic showers initiated by the iron primary of somewhat lower energy. However, the lateral spread of Cherenkov photons caused by the equilibrated QGP products is larger. All these effects depend on both the primary energy and the distance between detector and shower maximum; the latter observable is also energy dependent.

\section{Conclusions}

The lateral ground level Cherenkov photon densities of the extended air showers can be used to identify showers that follow the QGP transformation in the first interaction. It is worth noting that similar QGP related effects can be observed in lateral distributions of high momenta muons detected underground [5].

This work was supported by the Ministry of Education of the Czech Republic under contracts Nos. LN134 and LN00A006.

\section{REFERENCES}

1. S.P.Swordy et al., Astropart.Phys. 18 (2003) 129.

2. M.Gaźdicki, Z.Phys. C 66 (1995) 659.

3. J.D.Bjørken, Phys.Rev. D27 (1982) 140.

4. D.Heck et al., Report FZKA 6019 (1998).

5. J.Ř́idký, Astropart.Phys. 17 (2002) 355. 